\title{
LIN-23, an E3 Ubiquitin Ligase Component, Is Required for the Repression of CDC-25.2 Activity during Intestinal Development in Caenorhabditis elegans
}

\author{
Miseol Son ${ }^{1}$, Ichiro Kawasaki ${ }^{1}$, Bong-Kyeong $\mathrm{Oh}^{2}$, and Yhong-Hee Shim ${ }^{1, *}$
}

\begin{abstract}
Caenorhabditis elegans (C. elegans) utilizes two different cell-cycle modes, binucleations during the L1 larval stage and endoreduplications at four larval moltings, for its postembryonic intestinal development. Previous genetic studies indicated that CDC-25.2 is specifically required for binucleations at the L1 larval stage and is repressed before endoreduplications. Furthermore, LIN-23, the $C$. elegans $\beta$-TrCP ortholog, appears to function as a repressor of CDC-25.2 to prevent excess intestinal divisions. We previously reported that intestinal hyperplasia in lin-23(e1883) mutants was effectively suppressed by the RNAi depletion of cdc-25.2. Nevertheless, LIN-23 targeting CDC-25.2 for ubiquitination as a component of E3 ubiquitin ligase has not yet been tested. In this study, LIN-23 is shown to be the major E3 ubiquitin ligase component, recognizing CDC-25.2 to repress their activities for proper transition of cell-cycle modes during the $C$. elegans postembryonic intestinal development. In addition, for the first time that LIN-23 physically interacts with both CDC-25.1 and CDC-25.2 and facilitates ubiquitination for timely regulation of their activities during the intestinal development.
\end{abstract}

\section{INTRODUCTION}

Caenorhabditis elegans (C. elegans) is an ideal model organism to study cell cycle regulation during the development of multicellular organisms, owing to its invariant cell lineage and well defined cell anatomy (Deppe et al., 1978; Sulston and Horvitz, 1977; Sulston et al., 1983). The intestine is an essential organ for multiple biological processes including the distribution

${ }^{1}$ Department of Bioscience and Biotechnology, Konkuk University, Seoul 05029, Korea, ${ }^{2}$ Institute of Medical Science, Hanyang University College of Medicine, Seoul 04763, Korea

*Correspondence: yshim@konkuk.ac.kr

Received 10 October, 2016; revised 2 November, 2016; accepted 7 November, 2017; published online 18 November, 2016

Keywords: C. elegans, CDC-25.2, E3 ubiquitin ligase, intestinal development, LIN-23 of nutrition, immunity, stress response, and aging (An and Blackwell, 2003; Libina et al., 2003; Mallo et al., 2002; Sharrock, 1983). In C. elegans, the intestinal cells originate from a single embryonic blastomere, E, undergoing four rounds of nearly symmetrical cell divisions during the embryogenesis to produce 16 intestinal cells, termed the 16E cells (Deppe et al., 1978; Leung et al., 1999; Sulston et al., 1983). Subsequently, another round of cell divisions occurs in the two anterior and the two posterior intestinal cells. As a result, the intestine consists of 20 cells at the point of hatching (Leung et al., 1999; Sulston et al., 1983). After the hatching, intestinal cells adopt specialized division cycles. At the first (L1) larval stage, some, but not all, intestinal nuclei undergo nuclear divisions without cytokinesis, resulting in binucleated cells (Sulston and Horvitz, 1977), called as binucleation. As a result, worms in the late L1 stage possess 30 to 34 intestinal nuclei in the 20 cells. Thereafter, all the intestinal nuclei undergo endoreduplication before each larval molting to double their DNA contents without nuclear division or cytokinesis (Hedgecock and White, 1985). Because intestinal nuclei endoreduplicate four times up to the four larval stages, they finally contain 32n DNA contents per nucleus at the adult stage. In this way, the intestinal divisions in $C$. elegans change their cell cycle modes from cell divisions, binucleations to endoreduplications. However, the timely regulation of the transitions of these division modes still remains elusive.

Cdc25 phosphatase promotes cell cycle through dephosphorylation of cyclin-dependent kinases (Cdks) (Fantes, 1979). There are four cdc25 homologs in C. elegans; cdc-25.1, cdc-25.2, $c d c-25.3$, and cdc-25.4 (Ashcroft et al., 1998). Prolonged CDC25.1 activity in the cdc-25.1 gain-of-function (gf) mutants is reported and is usually required up to the $16 \mathrm{E}$ cell stage in wild type, caused extra intestinal cell divisions during the embryogenesis (Clucas et al., 2002; Kostić and Roy, 2002). The sequence analysis revealed that these $c d c-25.1$ (gf) mutants contained mutations in the conserved DSG $(X)_{2+n} S$ motif (Clucas et al., 2002; Kostić and Roy, 2002), which is recognized by a beta-transducin repeats-containing protein ( $\beta$-TrCP), the substrate recognition subunit of the $S \mathrm{CF}^{\beta-\mathrm{TrCP}} \mathrm{E} 3$ ubiquitin ligase, when both of the two serine (S) residues in the motif are phosphorylated (Fuchs et al., 2004). The SCF ${ }^{\beta-T r C P}$ E3 ubiquitin ligases play pivotal roles in cell-cycle regulation by targeting critical cellcycle regulators (Fuchs et al., 2004). Genetic studies suggest that the $g f$ mutations in the motif prevented the recognition of 
mutated CDC-25.1 by LIN-23, a C. elegans $\beta$-TrCP ortholog, which permitted the prolonged presence of the CDC-25.1 protein, resulting in intestinal hyperplasia in the cdc-25.1(gf) mutants (Hebeisen and Roy, 2008). In addition, CDC-25.2 is reported to be required for both the intestinal cell divisions after the $16 \mathrm{E}$ cell stage during the embryogenesis and binucleations during the L1 larval stage (Lee et al., 2016). We further revealed that the intestinal hyperplasia in lin-23(e1883) mutants was effectively suppressed by RNAi depletion of cdc-25.2 (Lee et al., 2016). This result suggests that LIN-23 targets CDC-25.2 for ubiquitination, and CDC-25.2 activity repressed by LIN-23 is required to change the intestinal cell-cycle mode from binucleation to endoreduplication. These findings indicate that timely repression of CDC-25.1 and CDC-25.2 activities by LIN-23 is essential for proper transitions of division modes during $C$. elegans intestinal development. Nevertheless, LIN-23, the C. elegans $\beta$-TrCP ortholog, indeed recognizes CDC-25.1 and CDC25.2 for ubiquitination has not yet been investigated. In this study, LIN-23 is demonstrated to exert most significant contribution to the transition from binucleation to endoreduplication among the $C$. elegans E3 ligase components. Moreover, we show that LIN-23 physically interacts with CDC-25.1 and CDC25.2, and facilitates their ubiquitination.

\section{MATERIALS AND METHODS}

\section{Caenorhabditis elegans strains}

Nematodes were cultured and handled at $20^{\circ} \mathrm{C}$ using standard methods (Brenner, 1974). C. elegans strains used in this study are as follows: N2 Bristol and MR156: rrls01[Pelt-2::GFP; unc$119(+)$ ] X, which were used as wild type. CB3514: lin23(e1883)/dpy-10(e128) II, YHS47: lin-23(e1883)/dpy-10(e128) II; rrls01[Pelt-2::GFP + unc-119(+)] X, DH2: zyg-11(b2) II. DS97: mat-2(ax76) II, EU593: mel-26(or184) I, EU640: cul2(or209) III, UP63: mat-3(ku233) III, NJ582: cul-1(e1756)/unc69(e587) III, VC141: zif-1(gk117) III, VC370: rfp-1(ok572)/eT1 III; +/eT1 V, VC562: rbx-1(ok782) V/nT1[q/s51] (IV;V), VC684: rbpl-1(ok907) I/hT2[bli-4(e937)let-?(q782)gls48] (I;III), VC954: rnf-113(ok1401) III/hT2[bli-4(e937) let-?(q782)q/s48] (I;III), VC1241: skr-1(ok1696) I, VC1439: skr-2(ok1938) I/hT2[bli4(e937) let-?(q782)qls48] (I;III), YHS171: rfp-1(ok572) III/hT2[bli-4(e937)let-?(q782)gls48] (I;III); rrls01[Pelt-2::GFP + unc-119(+)] X, YHS161: rbx-1(ok782) V/nT1[qls51] (IV;V); rrls01[Pelt-2::GFP + unc-119(+)]X, and YHS163: skr-1(ok1696) I; rrls01[Pelt-2::GFP + unc-119(+)]X.

\section{Quantification of intestinal nuclei and microscopy}

To examine the number of intestinal nuclei marked by GFP using an elt-2::GFP transgene, worms were transferred to 0.2 $\mathrm{mM}$ tetramisole in M9 buffer on a poly-L-lysine-coated slide glass, covered with a coverslip and observed using a fluorescence microscope (Zeiss Axioskop 2, Carl Zeiss, Germany). Otherwise, DNA of some strains, whose intestine is not marked with intestinal GFP, was stained with Hoechst 33342 solution (40 mM NaCl, $10 \mathrm{mM}$ Tris-HCl (pH7.5), 1 mM EDTA, 20\% Glycerol, $20 \mu \mathrm{g} / \mathrm{ml}$ Hoechst 33342). The worms were placed on a water drop on a poly-L-lysine-coated slide glass and fixed by quickly dehydrating on an alcohol lamp. The fixed worms were covered with a coverslip containing Hoechst 33342 solution and gelutol. The samples prepared on a slide glass were observed using a fluorescence microscope (Zeiss Axioskop 2, Carl Zeiss, Germany). Images were taken using an Orca-ERG digital camera (Hamamatsu, Japan) and NIS-elements software (Nikon, Japan).

\section{RNA interference}

RNAi depletion of $c d c-25.2$ in mutants of E3 ubiquitin-ligase complex genes was performed by the soaking RNAi method as previously described (Lee et al., 2016). The cdc-25.2 dsRNA was prepared by in vitro transcription using a cdc-25.2 cDNA clone, yk472b2, as the DNA template. Worms synchronized at the first larval (L1) stage were soaked into the dsRNA solution and incubated for $48 \mathrm{~h}$ at $20^{\circ} \mathrm{C}$. Then the soaked animals were transferred to OP50-seeded NGM plates and allowed to grow until the young adult stage. The efficiency of RNAi treatment was confirmed by the expression germ-line proliferation defective (Glp) phenotype, which is one of the characteristic cdc-25.2 RNAi phenotypes, and when confirmed, the number of intestinal nuclei was counted to determine the possible suppression of the mutant intestinal phenotype by cdc-25.2 RNAi.

\section{Cell culture}

HeLa cells were maintained in Dulbecco's Modified Eagle's Medium (Welgene, Korea), supplemented with $10 \%$ fetal bovine serum (Welgene), $100 \mathrm{U} / \mathrm{ml}$ penicillin (Welgene), and 100 $\mu \mathrm{g} / \mathrm{mL}$ streptomycin (Welgene) in a humidified atmosphere with $5 \% \mathrm{CO}_{2}$ at $37^{\circ} \mathrm{C}$.

Plasmid construction and transfection

To generate Myc-tagged CDC-25.1 and CDC-25.2 fusion proteins at their N-terminal, cDNA sequences of $c d c-25.1$ and $c d c-$ 25.2 were cloned into the pCMV-Myc vector (Clontech, USA). The cDNAs of $c d c-25.1$ and $c d c-25.2$ were PCR-amplified from a $C$. elegans mixed-stage cDNA library by using the following primer pairs: 5'-ggtacc ATG GCT ACC ACC GGG GAA AAA G-3' and 5'-gcggccgc TTA TTC GGC GTC GTC AGA AAT C-3' for cdc-25.1 cDNA; 5'-gg ggtacc ATG AAT AGG CCT TCA CAA ATA TC-3' and 5'-attt gcggccgc TTA TGA AAA ACG AGG ACA TGT C-3' for cdc-25.2 cDNA. Kpnl and Notl cleavage sites underlined in the primer sequences were used for cloning. To express GFP-tagged LIN-23 in mammalian cells, lin-23 cDNA sequence was PCR-amplified from a C. elegans mixed-stage cDNA library using the following primer pair: $5^{\prime}$-gg ggtacc ATG TCT TCA CCG CAC CG-3' and 5'-tcc cccggg TTA TGG GCC ACC ATC TG-3'. Using the restriction sites of Kpnl and Smal underlined in the sequences, cDNA of lin-23 was ligated into the $\mathrm{pEGFP}-\mathrm{C} 1$ plasmid DNA (Clontech). The DNA sequence of the cloned plasmid construct was confirmed by sequencing (Macrogen, Korea). To construct FLAG-tagged LIN-23 plasmid, cDNA sequence of lin-23 was cloned into the pCMV-Myc vector (Clontech). Using Myc-tagged LIN-23 plasmid as a template, inverse PCR was performed with primers: 5'-ATG GAC TAC AAG GAC GAC GAT GAC AAG GGT ACC ATG TCT TCA CCG CAC-3' and 5'-GT CCT TGT AGT CCA TGG TGG GCC CGC GGG TAC-3'. The Myc sequence in the Myc-tagged LIN-23 plasmid was replaced with FLAG sequence using In-Fusion HD cloning kit (Clontech) to generate FLAGtagged LIN-23 plasmid. The HA-tagged ubiquitin under the control of the CMV promoter was generated as previously described (Yoo et al., 2014). HeLa cells were transfected with 1 $\mu \mathrm{g}$ of each plasmid DNA using jetPRIME reagent (polyplus, France), following the manufacturer protocol. Same amount of empty vector was transfected to negative control samples as a loading control of the transfection. At $48 \mathrm{~h}$ after the transfection, the cells were harvested for further analyses.

\section{Co-immunoprecipitation and Western blot analysis}

Cell pellets were resuspended in NP-40 lysis buffer $(150 \mathrm{mM}$ $\mathrm{NaCl}, 1.0 \% \mathrm{NP}-40,50 \mathrm{mM}$ Tris-Cl, $\mathrm{pH}$ 8.0) containing protease 
inhibitor mixture (Roche Applied Bioscience, Germany) and incubated for $30 \mathrm{~min}$ at $4^{\circ} \mathrm{C}$, and sonicated (CosmoBio, Japan). Then, $650 \mu \mathrm{g}$ of cell lysates were incubated with protein Gsepharose (Sigma, USA) at $4^{\circ} \mathrm{C}$ overnight, and $2 \mu \mathrm{g}$ of monoclonal anti-c-Myc antibody (9E10, Santa Cruz, USA) was added to the samples. The samples were incubated for $4 \mathrm{~h}$ at $4^{\circ} \mathrm{C}$ with gentle rotation. After washing with NP-40 lysis buffer 4 times, samples were boiled in sodium dodecyl sulfate (SDS) loading buffer for $10 \mathrm{~min}$. Boiled cell lysate proteins were separated by $7-10 \%$ SDS-PAGE and transferred to a polyvinylidene difluoride membrane (Millipore, USA) using a semidry transfer apparatus (Hoefer, USA). The membrane was blocked with $5 \%$ nonfat dried milk/2\% BSA in TBS-T (150 mM NaCl, $50 \mathrm{mM}$ Tris-Cl, $0.1 \%$ Tween 20 ) for 30 min at RT. The blots on the membrane were first incubated with the following primary antibodies at $4^{\circ} \mathrm{C}$ overnight: anti-HA antibody (1:1,000, Y-11, Santa Cruz); antiMyc antibody (1:1,000, 9E10, Santa Cruz); anti-GFP antibody (1:1,000, A11122, Invitrogen, USA); monoclonal anti-FLAG M2 antibody (1:500, F1804, Sigma); monoclonal anti- $\alpha$-Tubulin antibody (1:5,000, T9026, Sigma). The blots on the membrane were then incubated with the secondary antibody, horseradish peroxidase-conjugated anti-mouse $\lg$ (1:5,000, Santa Cruz) or horseradish peroxidase-conjugated anti-rabbit IgG $(1: 5,000$, Santa Cruz), at RT for 2 h. Finally, immunoreactive blots were detected as chemiluminescence signals by using ECL reagent (Amersham ${ }^{\circledR}$ Biotechnology, UK) and a Bio-imaging Analyzer LAS-4000 (Fuji-film, Japan)

\section{In vivo ubiquitination assay}

HeLa cells were co-transfected with plasmid DNAs expressing HA-tagged ubiquitin, Myc-tagged CDC-25.1 or CDC-25.2, and GFP-tagged LIN-23 for $48 \mathrm{~h}$ and then treated with $25 \mu \mathrm{M}$ of MG132 (Sigma) for $6 \mathrm{~h}$ to inhibit the activity of proteasome and then harvested. The harvested cell pellets were then lysed in the NP-40 lysis buffer with protease inhibitors as previously described. Myc-tagged CDC-25.1 or Myc-tagged CDC-25.2 was immunoprecipitated with anti-Myc antibody (9E10, Santa Cruz) and protein $\mathrm{G}$ sepharose beads (Sigma). The immunoprecipitates were washed four times with NP-40 lysis buffer and boiled in SDS loading buffer for $10 \mathrm{~min}$. The immunoprecipitated and boiled samples were then analyzed by Western blot analysis.

\section{Statistical analysis}

The bar graphs are shown as the average \pm S.D. Box-andwhisker plots are displayed using Tukey whiskers, extending up to data points 1.5 interquartile ranges away from the first and the third quartile. $P$ value was calculated by Student's $t$-test to identify statistically significant difference. A $P$-value of $<0.05$ was considered significant. Most data were obtained from more than three independent experiments with multiple sample numbers $(n)$, indicated in the figure legends.

\section{RESULTS}

Extra intestinal nuclear divisions were observed in several mutants of E3 ubiquitin-ligase component genes, but the highest number of intestinal nuclei was observed in lin-23 mutants

In a previous genome-wide RNAi screen of ubiquitinationrelated genes, RNAi depletion of 17 genes induced excess intestinal cell divisions during the embryogenesis (Du et al., 2015). Among the 17 genes, lin-23 was included. We previously reported that intestinal hyperplasia in lin-23(e1883) mutants
Table 1. Mutant alleles of $C$. elegans E3 ubiquitin ligase-component genes examined in this study

\begin{tabular}{llll}
\hline Gene & Allele & $\begin{array}{l}\text { Class of E3 ligase } \\
\text { complex }\end{array}$ & Reference \\
\hline lin-23 & e1883 & CRL1/SCF (F-box) & Kipreos et al., 2000 \\
cul-1 & e1756 & CRL1/SCF (Cullin) & Kipreos et al., 1996 \\
cul-2 & or209 & CRL2 (Cullin) & Burger et al., 2013 \\
skr-1 & ok1696 & CRL1/SCF (Skp1) & Nayak et al., 2002 \\
skr-2 & ok1938 & CRL1/SCF (Skp1) & Nayak et al., 2002 \\
rbx-1 & ok782 & CRL1, 2, 3 & Kamura et al., 1999 \\
zyg-11 & b2 & CRL2 (VHL) & Sonneville and \\
& & & Gönczy, 2004 \\
zif-1 & gk117 & CRL2 (SOCS-box) & DeRenzo et al., 2003 \\
mel-26 & or184 & CRL2 (BTB) & Pintard et al., 2003 \\
mat-2 & ax76 & APC/C & Golden et al., 2000 \\
mat-3 & $k$ ku233 & APC/C & Golden et al., 2000 \\
rnf-113 & ok1401 & RING-type & Lee et al., 2013 \\
rbpl-1 & ok907 & RING-type & Huang et al., 2013 \\
rfp-1 & ok572 & RING-type & Crowe and Candido, \\
& & & 2004 \\
\hline
\end{tabular}

was effectively suppressed by the RNAi depletion of $c d c-25.2$ (Lee et al., 2016). We hypothesized that if the activity of CDC25.2 is negatively regulated not only by LIN-23 but also by other E3 ubiquitin ligase components during the postembryonic intestinal development, loss-of-function (If) mutants of these genes would display excess intestinal nuclear division phenotype during the larval development, and further this excess intestinal nuclear division phenotype would be suppressed by RNAi depletion of $c d c-25.2$ as seen in lin-23(e1883) mutants. Therefore, first we examined the number of intestinal nuclei in the If mutants of the ubiquitination-related genes at their adult stage (Fig. 1). Among the 17 genes, RNAi depletion previously reported to show excess intestinal cells (Du et al., 2015), 14 genes were tested, because their If mutant strains were available from the Caenorhabditis Genetic Center (Table. 1 and Fig. 1A). Among them, for cul-1(e1756), skr-2(ok1938), rbx-1(ok782), rnf113(ok1401), and rbpl-1(ok907), their heterozygous mutant animals were examined instead of homozygous mutant ones (Fig. 1A), because their homozygous animals could not develop to the adult stage. They showed a normal number of intestinal nuclei compared to the wild type control, suggesting that these genes are haplosufficient in the intestinal development (Fig. 1A). Among the 14 ubiquitination-related genes, lin23(e1883), cul-2(or209), mat-2(ax76), mat-3(ku233), and rfp1(ok572) homozygous if mutant animals showed statistically significant increase in the number of intestinal nuclei compared to the wild type (Fig. 1A). For example, immunofluorescent microscopic images of GFP-marked intestinal nuclei in wildtype, lin-23(e1883), skr-1(ok1696), and rfp-1(ok572) mutant adult animals are shown in Fig. 1B. Notably, although statistically significant, the increase in the number of intestinal nuclei in the cul-2, mat-2, mat-3, and rfp-1 If mutants was much less than those in lin-23(e1883) mutants. The four mutants contained only 1 to 5 more intestinal nuclei than the wild type, 


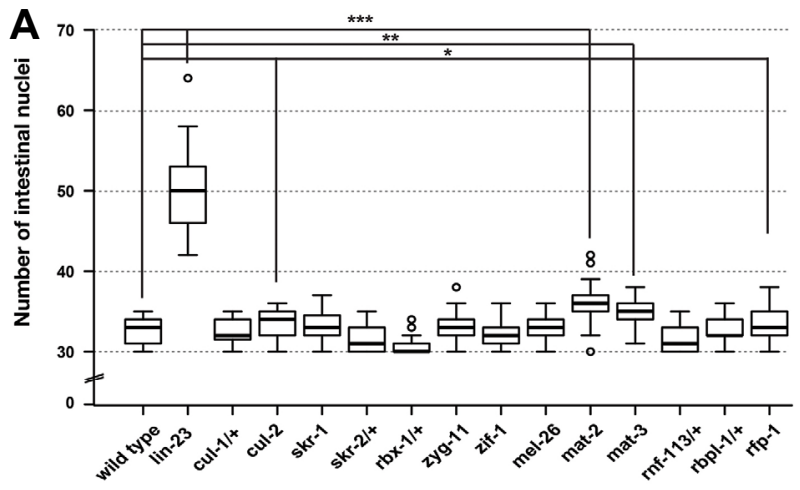

B

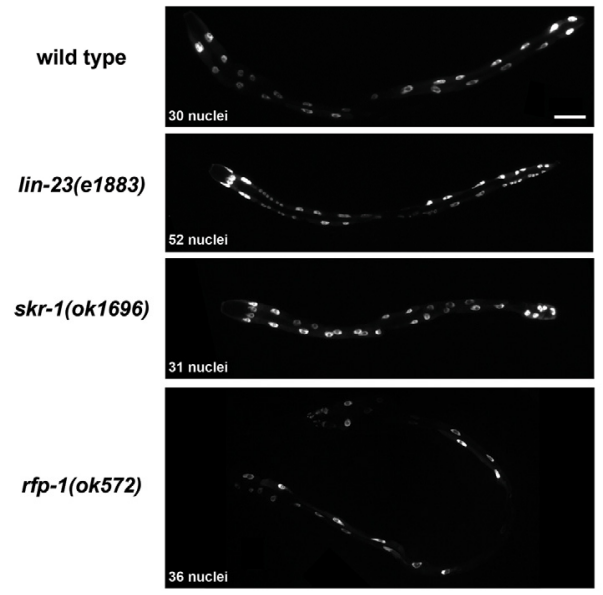

Fig. 1. Extra intestinal nuclear division phenotype was most prominent in lin-23 mutants among the mutants of E3 ubiquitin-ligase component genes. (A) Numbers of intestinal nuclei at the adult stage in mutants of E3 ubiquitin-ligase component genes, displayed as box-and-whisker plots. In the plots, median, $25 \%$ quartile, and $75 \%$ quartile are shown as a thick middle line, a lower edge, and an upper edge of a box, respectively. The ends of whiskers indicate the lowest and the highest data within 1.5 interquartile ranges from the $25 \%$ quartile and the $75 \%$ quartile, respectively (N2, n = 33; lin23, $\mathrm{n}=13$; cul-1, $\mathrm{n}=35$; cul-2, $\mathrm{n}=22$; skr-1, $\mathrm{n}=19$; skr-2, $\mathrm{n}=26$; rbx-1, $\mathrm{n}=25 ;$ zyg-11, $\mathrm{n}=17 ;$ zif-1, $\mathrm{n}=19 ;$ mel-26, $\mathrm{n}=49 ;$ mat-2, $\mathrm{n}$ $=35 ;$ mat-3, $\mathrm{n}=25 ;$ rnf-113, $\mathrm{n}=24 ;$ rbpl-1, $\mathrm{n}=25 ;$ rfp-1, $\mathrm{n}=26)$. * $p$ $<0.05 ;{ }^{* *} p<0.00005 ;{ }^{* * *} p<0.0000001$; the remains did not show statistically significant increment of number of intestinal nuclei, i.e., $p$ $\geq 0.05$. (B) Images of the intestinal nuclei at the adult stage in the wild type and respective mutants, visualized by the expression of elt-2-promoter driven GFP. Upper left, the anterior side. Scale bars, $50 \mu \mathrm{m}$.

whereas lin-23(e1883) mutants contained around 17 more intestinal nuclei than the wild type (Fig. 1A). Therefore, LIN-23 was considered as the major E3 ubiquitin ligase component that represses the CDC-25.2 activity during the postembryonic intestinal development.

RNAi depletion of cdc-25.2 suppressed extra intestinal nuclear divisions in mutants of E3 ubiquitin-ligase component genes

In lin-23(e1883) mutants, additional intestinal nuclear divisions occur in the place of endoreduplication at the L1 to L2 molting

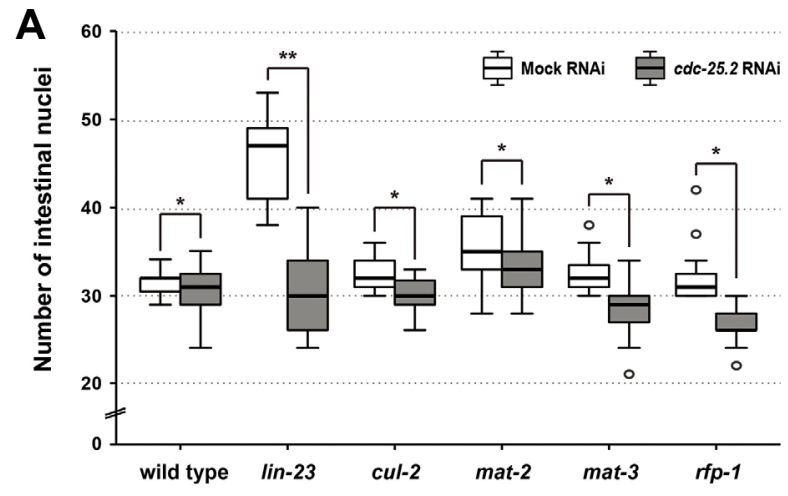

B

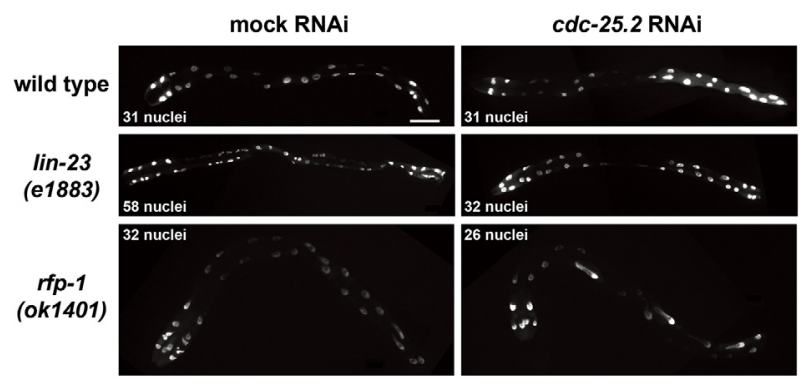

Fig. 2. Extra intestinal nuclear division phenotype was most significantly suppressed by cdc-25.2 RNAi in lin-23 mutants among the mutants of E3 ubiquitin-ligase component genes. RNAi depletion of cdc-25.2 was performed when the worms were at the first larval stage, and the numbers of intestinal nuclei were monitored when the RNAi-treated worms were grown to the adult stage. (A) Boxand-whisker plots show the numbers of intestinal nuclei in E3 ubiquitin-ligase component gene mutants without (white boxes, wild type, $\mathrm{n}=37$; lin-23, $\mathrm{n}=9$; cul-2, $\mathrm{n}=33$; mat-2, $\mathrm{n}=48$; mat $-3, \mathrm{n}=$ $47 ; r f-1, n=19$ ) or with (gray boxes, wild type, $n=39$; lin-23, $n=9$; cul-2, $\mathrm{n}=22 ;$ mat-2, $\mathrm{n}=38 ;$ mat-3, $\mathrm{n}=64 ;$ rfp-1, $\mathrm{n}=17$ ) cdc-25.2 RNAi treatment. ${ }^{*} p<0.05$; ${ }^{* *} p<0.00005$. (B) Images of the intestinal nuclei expressing elt-2-promoter driven GFP in mock or cdc25.2 RNAi-treated wild-type and E3 ubiquitin-ligase mutant adult worms. Left, the anterior side. Scale bars, $50 \mu \mathrm{m}$.

(Kipreos et al., 2000). Furthermore, this intestinal hyperplasia in the lin-23 mutants was almost completely suppressed by the RNAi depletion of $c d c-25.2$ (Lee et al., 2016). Because intestinal hyperplasia was also observed in If mutants of several E3 ubiquitin-ligase component genes besides the lin-23 mutants (Fig. 1), we hypothesized that these E3 ubiquitin-ligase components may also repress the CDC-25.2 activity to shift the intestinal division mode from nuclear division to endoreduplication. In such case, intestinal hyperplasia phenotype in these If mutants would be suppressed by the RNAi depletion of cdc-25.2, as observed for the lin-23 mutants. To test this possibility, we treated RNAi depletion of cdc-25.2 to the If mutants of cul-2, mat-2, mat-3, and $r f p-1$ to investigate whether their intestinal hyperplasia phenotype was suppressed (Fig. 2). cdc-25.2 RNAi indeed found to suppress the intestinal hyperplasia phenotype of the mutants of cul-2, mat-2, mat-3, and $r f p-1$ at statistically significant levels (Figs. $2 \mathrm{~A}$ and $2 \mathrm{~B}$ ). However, their levels of suppression $(p<0.05)$ were statistically less significant than the level of suppression observed in lin-23(e1883) mutants ( $p<$ 


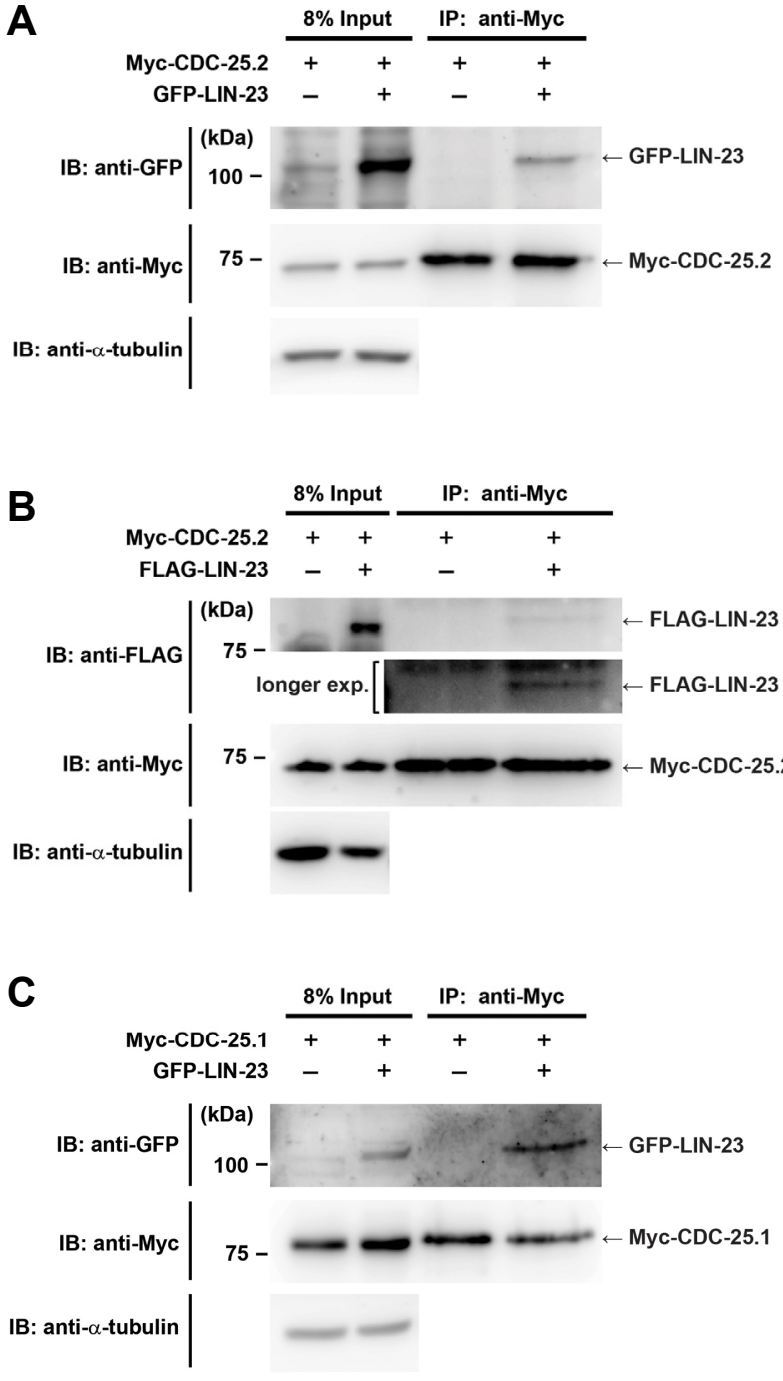

Fig. 3. CDC-25.2 and CDC-25.1 physically interacted with LIN-23. (A) Myc-tagged CDC-25.2 with or without GFP-tagged LIN-23, (B) Myc-tagged CDC-25.2 with or without FLAG-tagged LIN-23, and (C) Myc-tagged CDC-25.1 with or without GFP-tagged LIN-23, were expressed respectively in HeLa cells. Transfected cell lysates were immunoprecipitated (IP) with anti-Myc antibody. Input cell lysates $(8 \%)$ and immunoprecipitated products were immunoblotted (IB) with antibodies against GFP, Myc, and a-tubulin, respectively. $\alpha$-tubulin was used as an internal control of input cell lysates. Molecular sizes are indicated as kilodaltons $(\mathrm{kDa})$ on the left. Positions of respective proteins are indicated on the right.

$0.00005)$. These results support our view that LIN-23 is the major E3 ubiquitin ligase component that represses the CDC25.2 activity during the postembryonic intestinal development, although other E3 ubiquitin ligase components may also participate in this repression.

\section{CDC-25.2 and CDC-25.1 interact with LIN-23}

To examine whether LIN-23, a C. elegans $\beta$-TrCP ortholog, physically interacts with $\mathrm{CDC}-25.2$, an in vivo mammalian cell culture system was used. HeLa cells were co-transfected with Myc-tagged CDC-25.2 and GFP-tagged LIN-23 (Fig. 3A), or with Myc-tagged CDC-25.2 and FLAG-tagged LIN-23 (Fig. 3B). Then, immunoprecipitation was performed using anti-Myc antibody. The immunoprecipitates were analyzed by western immunoblotting with either anti-GFP antibody (Fig. 3A) or antiFLAG antibody (Fig. 3B) to investigate the co-immunoprecipitation of GFP or FLAG-tagged LIN-23 with Myc-CDC-25.2 proteins. Previous genetic studies in cdc-25.1(gf) mutants suggested that a mutation of $\beta$-TrCP motif causes intestinal hyperplasia, and the possibility that LIN-23 targets the CDC-25.1 by recognizing the $\beta$-TrCP concensus sequence was proposed (Hebeisen and Roy, 2008). However, there is no molecular evidence for the interaction between CDC-25.1 and LIN-23. Therefore, in this study, the interaction between CDC25.1 and LIN-23 was also analyzed. GFP-tagged LIN-23 was also co-immunoprecipitated with Myc-CDC-25.1 (Fig. 3C). The immunoprecipitates were also analyzed with anti-Myc antibody to check the efficiency of immunoprecipitation. In both the CDC-25.1 and CDC-25.2 immunoprecipitates, GFP-LIN-23 protein band of $\sim 100 \mathrm{kDa}$ in size (Figs. $3 A$ and $3 C$ ), or FLAG-LIN-23 protein band of $\sim 80$ $\mathrm{kDa}$ in size (Fig. $3 \mathrm{~B}$ ) was successfully detected (Figs. 3A-3C; the rightmost lanes). Input cell lysates were also examined, confirming the expression of respective recombinant proteins. These results indicate that, although CDC-25.2 does not contain a $\beta$-TrCP recognition consensus motif $\left(\mathrm{DSG}(\mathrm{X})_{2+n} \mathrm{~S}\right.$; see Discussion), both CDC-25.1 and CDC-25.2 physically interacted with LIN-23 in the mammalian cell culture system.

LIN-23 facilitated ubiquitination of CDC-25.1 and CDC-25.2 To examine whether the observed physical interaction between LIN-23 and CDC-25.1 and that between LIN-23 and CDC-25.2 actually increased the ubiquitination of CDC-25.1 and CDC25.2, respectively, an in vivo ubiquitination assay in HeLa cells was performed (Fig. 4). HeLa cells were transfected with several plasmid DNAs to express HA-tagged ubiquitin (Ub), Myctagged CDC-25.1 or Myc-tagged CDC-25.2, and GFP-tagged LIN-23. Then, the transfected HeLa cells were treated with, a proteasome inhibitor, MG132, to avoid the degradation of polyubiquitinated proteins (see "Materials and Methods" for details). Further, cell lysates prepared from the transfected cells were immunoprecipitated with anti-Myc antibody. Finally, the anti-Myc immunoprecipitates were western immunoblotted with anti-HA antibody to evaluate the levels of ubiquitination of respective immunoprecipitates. HA-positive ladder-like bands of various sizes were detected, which are indicative of polyubiquitination of proteins, in all the anti-Myc immunoprecipitates (Figs. 4A and $4 C$ ). The intensity of HA-positive ubiquitination signals was significantly stronger when GFP-LIN-23 was co-expressed in the cells compared to the cells, in which GFP-LIN-23 was not co-expressed (Figs. 4A and 4C). In the presence of GFP-LIN23, the relative intensities of HA-positive ubiquitination signals, which were normalized with those of Myc-positive signals, were about three times stronger in the Myc-CDC-25.1 immunoprecipitates, and about twice stronger in the Myc-CDC-25.2 immunoprecipitates, than in the absence of GFP-LIN-23, in which GFP was co-expressed as a mock (Figs. 4B and 4D). These results indicate that LIN-23 facilitated ubiquitination of CDC25.1 and CDC-25.2 in the mammalian cell culture system.

\section{DISCUSSION}

C. elegans utilizes three different cell-cycle modes for its intestinal development. First, normal cell divisions occur during the embryogenesis to generate 20 intestinal cells (Deppe et al., 1978; Leung et al., 1999; Sulston et al., 1983). Second, binucleations, the nuclear divisions without cytokinesis, occur during 


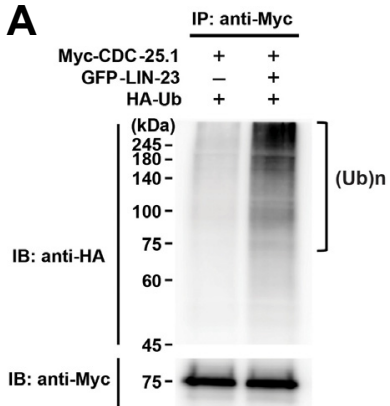

B

C

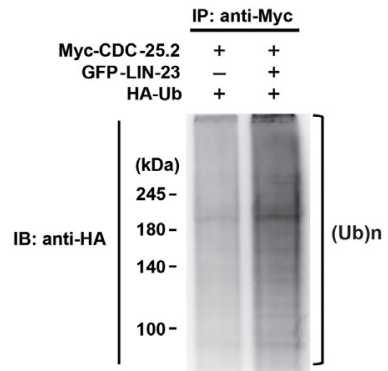

IB: anti-Myc $\quad 75-$

Fig. 4. LIN-23 facilitated ubiquitination of CDC-25.1 and CDC-25.2. In vivo ubiquitination of (A, B) CDC-25.1 and (C, D) CDC-25.2 by LIN-23. HeLa cells were transfected with HA-tagged ubiquitin and Myc-tagged CDC-25.1 (A) or Myc-tagged CDC-25.2 (C) with or without GFP-tagged LIN-23. MG132 was treated for $6 \mathrm{~h}$ before harvesting the transfected cell lysates to inhibit the activity of proteasome. The cell lysates were immunoprecipitated (IP) with antiMyc antibody and signals were detected by immunoblotting (IB) with antibodies against $\mathrm{HA}$ and Myc, respectively. The bottom panels show that similar amounts of Myc-tagged CDC-25.1 (A) or Myctagged CDC25.2 (C) were immunoprecipitated with or without GFPtagged LIN-23. Molecular sizes are indicated as kilodaltons $(\mathrm{kDa})$ on the left. (B, D) Relative ubiquitination levels of CDC-25.1 (B) and CDC-25.2 (D) either in the presence of GFP (negative control, represented as 1) or in the presence of GFP-tagged LIN-23. The intensity of HA signals within the bracket areas marked with $(\mathrm{Ub}) \mathrm{n}$ was measured and normalized with the intensity of corresponding Myc signals. The values shown in $B$ and $D$ were ratios of the levels of ubiquitination in GFP-LIN-23-transfected cells relative to those in the negative control, GFP-transfected cells. Error bars indicate the S. D. from three (B) or four (D) independent experiments. ${ }^{*} p<0.05$.

the L1 larval stage to produce 30-34 intestinal nuclei in the 20 cells (Sulston and Horvitz, 1977). Finally, endoreduplications, the DNA replications without nuclear divisions, occur in each intestinal nucleus at respective four larval moltings to increase their DNA contents. Previous genetic studies indicated that CDC-25.1 is specifically required for embryonic intestinal cell divisions up to the $16 \mathrm{E}$ cell stage, and then CDC-25.2 takes over the role of CDC-25.1 up to the stage of binucleations at the L1 larval stage, resulting in 30-34 nuclei in 20 intestinal cells (Lee et al., 2016). Furthermore, LIN-23, the $C$. elegans $\beta$-TrCP ortholog, was genetically identified as a repressor of CDC-25.1 and CDC-25.2 to prevent excess embryonic intestinal cell divisions and excess larval intestinal nuclear divisions, respectively (Hebeisen and Roy, 2008; Lee et al., 2016). Nevertheless, whether LIN-23 actually targets CDC-25.1 and CDC-25.2 for ubiquitination as a component of E3 ubiquitin ligase has not been investigated previously. In this study, by using an in vivo mammalian cell culture co-transfection system, for the first time, we demonstrated that LIN-23 physically interacts with CDC25.1 and CDC-25.2, and facilitates their ubiquitination.

$\mathrm{LIN-23}$ is a $C$. elegans ortholog of $\beta$-TrCP, a class of F-box protein, functioning as a substrate-recognizing subunit in the SCF (Skp1-Cullin-F-box protein) E3 ubiquitin ligase complex (Kipreos et al., 2000). In the complex, Cullin offers the scaffold to the complex and Skp1 serves as an adaptor protein that links Cullin and an F-box protein. $\beta$-TrCP interacts with a substrate that is phosphorylated within the $\mathrm{DSG}(\mathrm{X})_{2+n} \mathrm{~S}$ destruction motif (Maniatis, 1999). The SCF ${ }^{\beta-T r C P}$ E3 ubiquitin ligase mediates the ubiquitination and proteasomal degradation of the phosphorylated substrates that play a key role in the signal transduction or cell-cycle regulation (Fuchs et al., 2004). Previous studies revealed that $c d c-25.1(g f)$ mutants contained mutations in the conserved DSG $(X)_{2+n}$ S destruction motif (Clucas et al., 2002; Kostić and Roy, 2002). These findings strongly support the idea that, in wild type, CDC-25.1 protein is indeed targeted by LIN23-containing E3 ubiquitin ligase for ubiquitination and proteasomal degradation. In contrast, CDC-25.2 protein does not contain an amino-acid sequence that is completely matching to the $\mathrm{DSG}(\mathrm{X})_{2+n} \mathrm{~S}$ destruction motif sequence. Nevertheless, in this study, we found that GFP-LIN-23 or FLAG-LIN-23 was specifically co-immunoprecipitated with Myc-CDC-25.2 as with Myc-CDC-25.1, and that the ubiquitination of Myc-CDC-25.2 significantly increased in the presence of GFP-LIN-23 as that of Myc-CDC-25.1 in an in vivo mammalian cell culture system. Therefore, CDC-25.2 was assumed to be recognized by LIN-23 either using a sequence that does not completely match to the destruction motif or by an unidentified mechanism.

A previous bioinformatics study revealed 866 potential ubiquitination-related genes in C. elegans genome, including 836 E3 ubiquitin ligase component genes (Du et al., 2015). Through a systematic RNAi screening covering $80 \%$ of these potential ubiquitination-related genes, the above study identified 17 genes, RNAi of which induced excess intestinal cell divisions during the embryogenesis (Du et al., 2015). This finding suggests that these 17 gene products potentially function as repressors of embryonic intestinal cell divisions by targeting a critical cell-cycle regulator such as CDC-25.1 for ubiquitination. Indeed, lin-23 was included among the 17 genes (Du et al., 2015). In this study, to identify possible counterpart components that function together with LIN-23 in the same E3 ubiquitin ligase complex, or to find additional substrate-recognizing subunits that target CDC-25.2, 14 genes out of above 17 genes were tested to see whether viable If mutants of them also show excess intestinal nuclear divisions during the larval development. Among the Cullin and Skp1 ortholog genes, cul-2 homozygous mutants showed increased numbers of intestinal nuclei. Nevertheless, the level of increase was not so significant compared to that in lin-23 homozygous mutants. In contrast, cul-1 and skr-2 homozygous mutants could not be examined, because they could not develop to the adult stage. Furthermore, their heterozygous mutants did not show any significant difference in the number of intestinal nuclei from wild-type control. Therefore, counterpart components that likely function together with LIN-23 in the same E3 ubiquitin ligase complex could not be identified. Among the ortholog genes that encode components of different types of E3 ubiquitin ligase, mat-2, mat-3, and $r f p-1$ mutants showed increased numbers of intestinal nuclei. mat-2 and mat-3 encode the components of Anaphase- 
Promoting Complex (APC/C), the E3 ubiquitin ligase that has a critical role in cell-cycle regulation (Golden et al., 2000). rfp-1 encodes a component of RING-type E3 ligase (Crowe and Candido, 2004). Nevertheless, in these three mutants, the levels of increase in the number of intestinal nuclei and the levels of suppression of the number of intestinal nuclei by $c d c-25.2$ RNAi were also not as significant as those in lin-23 mutants. Therefore, we concluded that LIN-23 is the major E3 ubiquitin ligase component that recognizes both CDC-25.1 and CDC25.2 to repress their activities for proper transition of cell-cycle modes during the $C$. elegans intestinal development, although other E3 ubiquitin ligase components may also participate in this regulation.

\section{ACKNOWLEDGMENTS}

Some $C$. elegans strains used in this study were provided by the Caenorhabditis Genetic Center, which is supported by the National Institutes of Health, Office of Research Infrastructure Programs (P40 OD010440) and by the C. elegans Reverse Genetics Core Facility at the University of British Columbia, which is part of the international C. elegans Gene Knockout Consortium. We thank Dr. Yuji Kohara (Mishima, Japan) for the yk EST clones. This research was supported by the Basic Science Research Program through the National Research Foundation of Korea funded by the Ministry of Education [grant numbers 2013R1A1A2009090 and 2015R1D1A1A01057488 to Y.-H.S., 2015R1D1A1A01057853 to I.K.].

\section{REFERENCES}

Ashcroft, N.R., Kosinski, M.E., Wickramasinghe, D., Donovan, P.J., and Golden, A. (1998). The four cdc25 genes from the nematode Caenorhabditis elegans. Gene 214, 59-66.

An, J.H., and Blackwell, T.K. (2003). SKN-1 links C. elegans mesendodermal specification to a conserved oxidative stress response. Genes Dev. 17, 1882-1893.

Brenner, S. (1974). The Genetics of Caenorhabditis elegans. Genetics 77, 71-94

Burger, J., Merlet, J., Tavernier, N., Richaudeau, B., Arnold, A., Ciosk, R., Bowerman, B., and Pintard, L. (2013). CRL2 ${ }^{\text {LRR-1 }}$ E3Ligase Regulates Proliferation and Progression through Meiosis in the Caenorhabditis elegans Germline. PLoS Genet. 9, e1003375.

Clucas, C., Cabello, J., Büssing, I., Schnabel, R., and Johnstone, I.L. (2002). Oncogenic potential of a C. elegans cdc25 gene is demonstrated by a gain-of-function allele. EMBO J. 21, 665-674.

Crowe, E., and Candido, E.P. (2004). Characterization of C. elegans RING finger protein 1, a binding partner of ubiquitinconjugating enzyme 1. Dev. Biol. 265, 446-459.

Deppe, U., Schierenberg, E., Cole, T., Krieg, C., Schmitt, D., Yoder, B., and von Ehrenstein, G. (1978). Cell lineages of the embryo of the nematode Caenorhabditis elegans. Proc. Natl. Acad. Sci. USA 75, 376-380.

DeRenzo, C., Reese, K.J., and Seydoux, G. (2003). Exclusion of germ plasm proteins from somatic lineages by cullin-dependent degradation. Nature 424, 685-689.

Du, Z., He, F., Yu, Z., Bowerman, B., and Bao, Z. (2015). E3 ubiquitin ligases promote progression of differentiation during $C$. elegans embryogenesis. Dev. Biol. 398, 267-279.

Fantes, P. (1979). Epistatic gene interactions in the control of division in fission yeast. Nature 279, 428-430.

Fuchs, S.Y., Spiegelman, V.S., and Kumar, K.G.S. (2004). The many faces of beta-TrCP E3 ubiquitin ligases: reflections in the magic mirror of cancer. Oncogene 23, 2028-2036.

Golden, A., Sadler, P.L., Wallenfang, M.R., Schumacher, J.M.,
Hamill, D.R., Bates, G., Bowerman, B., Seydoux, G., and Shakes, D.C. (2000). Metaphase to anaphase (mat) transition-defective mutants in Caenorhabditis elegans. J Cell Biol. 151, 1469-1482.

Hebeisen, M., and Roy, R. (2008). CDC-25.1 stability is regulated by distinct domains to restrict cell division during embryogenesis in C. elegans. Development 135, 1259-1269.

Hedgecock, E.M., and White, J.G. (1985). Polyploid tissues in the nematode Caenorhabditis elegans. Dev. Biol. 107, 128-133.

Huang, P., Ma, X., Zhao, Y., and Miao, L. (2013). The C. elegans Homolog of RBBP6 (RBPL-1) Regulates Fertility through Controlling Cell Proliferation in the Germline and Nutrient Synthesis in the Intestine. PLoS One 8, e58736.

Kamura, T., Koepp, D.M., Conrad, M.N., Skowyra, D., Moreland, R.J., lliopoulos, O., Lane, W.S., Kaelin Jr., W.G., Elledge, S.J., Conaway, R.C. et al. (1999). Rbx1, a Component of the VHL Tumor Suppressor Complex and SCF Ubiquitin Ligase. Science 284, 657-661.

Kipreos, E.T., Lander, L.E., Wing, J.P., He, W.W., and Hedgecock, E.M. (1996). cul-1 is required for cell cycle exit in C. elegans and identifies a novel gene family. Cell 85, 829-839.

Kipreos, E.T., Gohel, S.P., and Hedgecock, E.M. (2000). The C. elegans F-box/WD-repeat protein LIN-23 functions to limit cell division during development. Development 127, 5071-5082.

Kostić, I., and Roy, R. (2002). Organ-specific cell division abnormalities caused by mutation in a general cell cycle regulator in C. elegans. Development 129, 2155-2165.

Lee, H., Alpi, A.F., Park, M.S., Rose, A., and Koo, H.S. (2013). C. elegans Ring Finger Protein RNF-113 Is Involved in Interstrand DNA Crosslink Repair and Interacts with a RAD51C Homolog. PLoS One 8, e60071.

Lee, Y.U., Son, M., Kim, J., Shim, Y.H., and Kawasaki, I. (2016). CDC-25.2, a C. elegans ortholog of $c d c 25$, is essential for the progression of intestinal divisions. Cell Cycle 15, 654-666.

Leung, B., Hermann, G.J., and Priess, J.R. (1999). Organogenesis of the Caenorhabditis elegans intestine. Dev. Biol. 216, 114-134.

Libina, N., Berman, J.R., and Kenyon, C. (2003). Tissue-specific activities of $C$. elegans DAF-16 in the regulation of lifespan. Cell 115, 489-502.

Mallo, G.V., Kurz, C.L., Couillault, C., Pujol, N., Granjeaud, S., Kohara, Y., and Ewbank, J.J. (2002). Inducible antibacterial defense system in C. elegans. Curr. Biol. 12, 1209-1214.

Maniatis, T. (1999). A ubiquitin ligase complex essential for the NF$\mathrm{kB}$, WntWingless, and Hedgehog signaling pathways. Genes Dev. 13, 505-510.

Nayak, S., Santiago, F.E., Jin, H., Lin, D., Schedl, T., and Kipreos, E.T. (2002). The Caenorhabditis elegans Skp1-related gene family: Diverse functions in cell proliferation, morphogenesis, and meiosis. Curr. Biol. 12, 277-287.

Pintard, L., Willis, J.H., Willems, A., Johnson, J.L., Srayko, M., Kurz, T., Glaser, S., Mains, P.E., Tyers, M., Bowerman, B. et al. (2003) The BTB protein MEL-26 is a substrate-specific adaptor of the CUL-3 ubiquitin-ligase. Nature 425, 311-316.

Sharrock, W.J. (1983). Yolk proteins of Caenorhabditis elegans. Dev. Biol. 96, 182-188.

Sonneville, R., and Gönczy, P. (2004). zyg-11 and cul-2 regulate progression through meiosis II and polarity establishment in $C$. elegans. Development 131, 3527-3543.

Sulston, J.E., and Horvitz, H.R. (1977). Post-embryonic cell lineages of the nematode, Caenorhabditis elegans. Dev. Biol. 56, 110-156.

Sulston, J.E., Schierenberg, E., White, J.G., and Thomson, J.N. (1983). The embryonic cell lineage of the nematode Caenorhabditis elegans. Dev. Biol. 100, 64-119.

Yoo, J.E., Park, Y.N., and Oh, B.K. (2014). PinX1, a telomere repeat-binding factor 1 (TRF1)-interacting protein, maintains telomere integrity by modulating TRF1 homeostasis, the process in which human telomerase reverse transcriptase (hTERT) plays dual roles. J. Biol. Chem. 289, 6886-6898. 